\title{
Semantic information modulates online morphological learning: neuromagnetic investigation
}

\author{
Viktória Roxána Balla ${ }^{1}$, Yury Shtyrov ${ }^{2}$, Lilli Kimppa ${ }^{1}$, Miika Leminen $^{3}$, Alina Leminen ${ }^{1,4}$ \\ ${ }^{1}$ Cognitive Brain Research Unit, Department of Psychology and Logopedics, Faculty of Medicine, \\ University of Helsinki, Finland \\ ${ }^{2}$ Department of Clinical Medicine, Center of Functionally Integrative Neuroscience, Aarhus \\ University, Aarhus, Denmark \\ ${ }^{3}$ AI and Analytics Development Unit, HUS Helsinki University Hospital, Helsinki, Finland \\ ${ }^{4}$ RDI, Laurea University of Applied Sciences, Vantaa, Finland
}

Corresponding author: Viktória Roxána Balla, Cognitive Brain Research Unit, Department of Psychology and Logopedics, Faculty of Medicine, University of Helsinki, PO box 21, (Haartmaninkatu 3), FI-00290 Helsinki, Finland. Phone: +358 0 2941911. Email: viktoria.balla@ helsinki.fi

Number of words: 7897

Number of figures: 4 


\begin{abstract}
:
Morphemes are defined as the smallest meaningful units of a language, which can be used to form new words or convey grammatical information. Learning the meaning of morphemes and recognizing morphemic boundaries is thus an essential part of language learning, however, neural mechanisms underlying the acquisition of morphemes remain largely unexplored. Here, we investigated the online build-up of memory traces for novel suffixes in the left perisylvian language network. Half of the novel suffixes were trained semantically through a picture-word association task and half were untrained. Following the semantic training with twenty repetitions per suffix, we used magnetoencephalography (MEG) to record cortical responses to the novel semantically trained, untrained, and real suffixes combined with real and pseudoword stems in a 20-minute-long repetitive exposure task. Participants were exposed to spoken stimuli passively, while their attention was diverted away from the auditory input. To assess the online learning, we compared neuromagnetic brain responses to the different suffixes in the beginning and the end of this passive exposure. Our results showed that semantically trained suffixes demonstrated a consistent activation pattern throughout the learning task, suggesting that repetitive exposure did not further facilitate learning after the formation of initial episodic memories during the semantic training. By contrast, neural source activation to untrained suffixes combined with real stems increased over the course of the passive exposure, reflecting a build-up of suffix-related memory traces. Online learning related changes were present in all regions of interest in the left hemisphere (inferior frontal gyrus, anterior temporal lobe, posterior superior temporal gyrus, posterior middle temporal gyrus). The distinction in the neural source activity patterns between the trained and untrained novel suffix types indicates that semantic information can give a head start to the suffix learning process. Furthermore, in the context of a real word stem, neural source activation elicited by both novel suffix types in the late phase of the passive exposure reached the early phase activation elicited by real suffixes. Our results suggest immediate learning effects to novel suffixes gained through a brief semantic learning as well as more incremental suffix learning effects obtained through purely perceptual exposure.
\end{abstract}

Keywords: morphology, suffix, semantics, rapid learning, magnetoencephalography 
The smallest building blocks of language that carry meaning are morphemes, which encode semantic or grammatical information (Gwilliams, 2020). Words can comprise one or more morphemes (e.g., stem + suffix, "dark + ness"), particularly in languages with rich morphology, such as Finnish, a striking case of extremely rich morphological structure. Monomorphemic words in Finnish account for only a small portion of words; in fact, the majority of Finnish words are polymorphemic and of a very low surface frequency, making derivation an integral part of word formation (Vannest et al., 2002). To fully master a language, whether it is the first language in childhood or a second language in adulthood, the ability to process morpheme constituents of complex words is crucial. Children start applying morphological endings relatively early in their speech (e.g., Stolt et al., 2009), and adult second-language learners can also develop sensitivity to morphological information (Kimppa et al., 2019; Steinhauer, 2014). However, very little is known about the neural underpinnings of the acquisition of morphological endings, particularly regarding derivational morphology.

With respect to the neural mechanisms underlying the processing of morphologically complex words, (regular) inflections have been shown to depend on left lateralized fronto-temporal networks, while derivations elicit bilateral processing (Bozic et al., 2010; Tyler et al., 2002; Whiting et al., 2013; for an extensive review see Leminen et al., 2018). Morphological decomposition has been shown to be mediated by the left inferior frontal gyrus, while temporal regions are more involved in lexico-semantic access (Marslen-Wilson \& Tyler, 2007). Importantly, recent electrophysiological findings have found no differences in the early responses ( $50 \mathrm{~ms}$ after the suffix onset) for inflected and derived words (Kimppa et al., 2019), supporting behavioral findings that both complex word types undergo early morphological decomposition (for reviews, see e.g., Amenta and Crepaldi, 2012, Rastle \& Davis, 2008).

While numerous models discuss the processing and storage of morphologically complex words, less research has focused on examining the underlying mechanisms of novel morpheme acquisition (Endress \& Hauser, 2011; Ferman et al., 2009; Ferman \& Karni, 2010; Havas et al., 2015, 2017; A. Leminen et al., 2016; M. Leminen et al., 2020; Merkx et al., 2011; Mirković et al., 2019; Mirković \& Gaskell, 2016; Nevat et al., 2017; Tamminen et al., 2012, 2015). Havas and colleagues (2015) reported behavioral evidence that adult language learners can quickly (after a 25-minute training session) acquire morphological information from a new language even when the morphological feature in question is absent in their native language (such as the meaning of morphemes denoting gender, a feature absent in Finnish). They found evidence of morphological decomposition of the to-be-learned words into the stem and suffix. The results were also taken to emphasize the importance of the learner's own native language in morphological processing. Namely, native speakers of morphologically more complex languages may apply continuous decomposition as their primary learning and processing strategy. Due to the abundance of suffixes in their native language, long-term experience in decomposition seems to be beneficial when it comes to identifying suffixes in a novel language (Havas et al., 2015, 2017; see also Prins et al., 2019).

Some studies point out that acquiring different aspects of novel morphology may rely on different memory systems, namely, declarative and procedural memory (Ferman et al., 2009; Nevat et al., 2017; Ullman, 2004, 2016). Ferman et al. (2009) investigated long-term acquisition of the phonological and semantic aspects of an artificial morphological rule (AMR) within a natural language over ten consecutive learning sessions with 1-3-day-long intervals of offline consolidation. The AMR contained a phonological transformation of Hebrew verbs undergoing a novel inflection, that was in line with the phonological constrains of the language. Applying the novel inflection required omitting the vowel preceding the added suffix as well as shifting the stress to the suffix. In addition, this novel inflection expressed a semantic distinction: the novel suffix added to the Hebrew verbs was dependent on the animacy of the preceding noun. Based on the participants' high accuracy (>75\%) in producing the phonological transformation (irrespective of semantic accuracy), the authors reported that, all participants generalized the phonological aspects of the rule within the first two sessions. This improvement was independent from explicit knowledge about the semantic aspect (i.e., consciously discovering the animacy rule). Remarkably, discovering the semantic distinction resulted in an abrupt enhancement of accuracy in both judgement and production tasks; this, however, required explicit learning and was not attained by all participants. On account of the independency of acquiring the phonological and semantic aspects of the morphological rule, Ferman and colleagues (2009) concluded that the two processes are supported by different contributions from the procedural and declarative memory systems, respectively (Ferman et al., 2009). Similarly, in a functional magnetic resonance 
imaging (fMRI) study, Nevat et al. (2017) investigated the learning and production of plural inflections using novel nouns and novel inflectional suffixes over three sessions with 3-4-day-long intervals inbetween. In each session, participants were trained to produce and judge the correctness of novel plural inflections, where the rhymes of words provided probabilistic cues to their suffix. During training, the meanings of novel nouns were illustrated with pictures. Inflection of both trained and untrained nouns was tested in the scanner at the end of the first and last sessions. Untrained nouns, which were first encountered during this test, contained the same probabilistic phonological cues to the correct suffix as trained items. Nevat and colleagues (2017) reported that inflecting trained items - which underwent extensive practice along with visual referents - activated the left pars triangularis, a region in the inferior-frontal cortex associated with recalling information from declarative memory (Ullman, 2004, 2006, 2016). However, generalizing these learned inflections to untrained nouns, relying only on phonological cues, elicited higher activation in brain structures associated with procedural memory (head of the caudate nucleus, pars opercularis, precentral gyrus, and the anterior part of the supplementary motor area), suggesting involvement of compositional processes. To summarize, the declarative and procedural memory systems may be involved in different aspects of the acquisition of novel morphology. Both studies propose that generalizing the phonological features of a newly learnt morphological rule recruits the procedural memory system.

Ferman et al. (2009) suggested that gaining explicit awareness of semantic features has a positive impact on the learning of novel morphology. The specific role of the introduction of semantic information versus mere phonological form on novel suffix acquisition has been investigated in several other behavioral studies, typically conducted in a setting involving offline consolidation. Following a single training session of novel derivational suffixes containing phonological form only or an additional novel meaning, Merkx and colleagues (2011) reported that participants successfully recognized both types of novel suffixes after two days of retention. This effect was demonstrated in their difficulty to reject previously not presented recombinant pairs of trained stems and suffixes in a recognition memory task (note that this result also suggests judgements based on morphemic instead of whole-form representations). However, in a lexical decision task, pseudowords that included the learnt suffixes with semantics took longer to reject than those with the novel form-only suffixes. This was interpreted to indicate that the development of lexicalized representations for novel suffixes benefited from not only a period of offline consolidation, but semantic information as well. This lexicalization effect was further corroborated by the participants' significantly higher than chance level performance in the definition selection accuracy for items containing untrained stems paired with semantically trained suffixes, implicating they could generalize the learned suffix semantics to novel combinations. Similarly, Tamminen et al. (2012) found an immediate processing advantage for semantically trained suffixes over untrained suffixes combined with familiar stems, indicated by significantly shorter reaction times in a speech shadowing task (Marslen-Wilson, 1985). However, the generalization of the suffix knowledge (both trained and untrained) to combinations with unfamiliar stems required an offline consolidation phase. However, in a series of experiments investigating the acquisition of novel semantically trained suffixes, Tamminen and colleagues (2015) showed that while generalization of a novel suffix in speeded tasks (i.e., when time pressure is introduced, in, e.g., semantic priming) requires a consolidation period, it may be disrupted if semantic inconsistency is introduced during learning. Although, in non-speeded tasks requiring slow, deliberate reasoning with little time pressure, groups tested both immediately and after a week's delay were able to make highly accurate explicit decisions about the sentential congruency of the novel suffix + unfamiliar stem combinations. While offline consolidation seems to play an important role in the formation of context-independent representations of suffixes, these results indicated that even immediate generalization is achievable given sufficient time to process the novel forms. Based on distinct immediate learning effects arising instantly after training and later, after a consolidation period seen in speeded versus non-speeded tasks, the authors suggested two different mechanisms involved in generalization: an online generalization, supported by overlapping representations emerging over a longer period of time in the neocortex, and offline generalization, supported by initial episodic memories mediated by the hippocampus. Thus, while the lexicalization of novel derivational suffixes is likely a longer process, initial learning effects arise immediately after one training session (Tamminen et al., 2015).

Immediate learning effects have also been identified in a study by Leminen and others (2016) that addressed the neural mechanisms of novel suffix acquisition in a morphologically rich language, 
Finnish, using MEG. They found that spoken complex forms with semantically trained novel derivational suffixes elicited stronger neural source activation in the left inferior frontal gyrus during passive exposure immediately after training compared to forms with novel suffixes without pre-trained meaning. This was suggested to reflect successful morphological decomposition of the semantically trained novel suffixes. While Leminen et al. (2016) did suggest that morphological decomposition might have taken place for novel suffixes distinguished by semantics already without long-term consolidation, they did not explore the unfolding neural changes over the course of the passive exposure to the novel suffixed forms. The passive repetitive exposure to the novel suffixed forms with derivational semantics vs. only phonological form may elicit distinct neural dynamics in different parts of the language network within shorter timescale than previously investigated. Additionally, direct comparisons of neural dynamics of newly learnt suffixes to those with real suffixed forms during earlystage acquisition are lacking. EEG studies have shown that short repetitive passive exposure to novel spoken monomorphemic word-forms elicited rapid activation enhancement in left inferior frontal and posterior temporal cortex (Kimppa et al., 2015; Shtyrov, 2011; Shtyrov et al., 2010; Yue et al., 2014). Such enhancement is suggested to indicate word memory-trace formation irrespective of attentional focus on the spoken input and was shown to correlate with behavioral measures of word learning (Kimppa et al., 2015). It is, however, yet to be determined whether such rapid changes in neural dynamics occur also for complex words with novel suffixes.

In the present study, we investigated the neural underpinnings of online memory trace formation for novel suffixes. We tracked the modulation of neuromagnetic activation to novel suffixes combined with real word and pseudoword stems, during passive repetitive exposure to the stimuli. Specifically, we addressed how acquisition of semantic information modulates the suffix-related memory trace formation. To this end, prior to the passive exposure session, the meaning of half of the novel suffixes was trained through a word-picture association task while the other half of the novel suffixes were not trained, they appeared only during the exposure session. Importantly, we also presented real suffixes paired with appropriate word stems, constituting derivational and inflectional forms, to compare the neural dynamics between existing and novel complex forms. Since all real suffixes were expected to undergo morphological decomposition (Amenta \& Crepaldi, 2012; Kimppa et al., 2019; Rastle \& Davis, 2008) and since neural source activity indeed did not differ significantly between the real diminutive suffix "-ke" (derivational) and the question clitic "-ko" (inflectional) in either time window of interest, to increase the signal-to-noise ratio, they were collapsed into the "real suffixes" category in our analyses (see Supplementary Material). Immediately after the semantic training, we recorded continuous magnetoencephalography (MEG) to novel suffixed trained and untrained forms and real suffixed forms during a short passive exposure. The high temporal resolution of MEG enabled precise extraction of neural activation related to the spoken stimuli.

In order to assess the neural activity associated with the learning dynamics of novel suffixes, we compared neural source activation to novel and real suffixes in the early and late phases of the passive exposure. As responses to familiar suffixes may be affected by repetition suppression effects over time (Bakker et al., 2013; De Grauwe et al., 2014; Hasting \& Kotz, 2008; Pulvermüller et al., 2008), we also contrasted the activation pattern to novel suffixes at the end of the exposure to that to the real suffixes in the beginning of the exposure to compare the strength of their memory traces. To assess the effects of explicit semantic training, we compared the neural dynamics between pre-trained and untrained suffixes in the early and late phases of the passive exposure. In order to assess the neuroanatomical structures underpinning any MEG effects, we performed distributed source analysis of the evoked neuromagnetic responses. We used a region of interest (ROI) analysis approach, to reduce the dimensionality and therefore, the number of comparisons performed on the data. Based on previous literature, we anticipated that learning-related activation dynamics for linguistic stimuli will be reflected specifically in the left inferior frontal gyrus (IFG) and temporal areas (anterior temporal lobe - ATL, posterior superior temporal gyrus - pSTG, posterior middle temporal gyrus - pMTG). The IFG has been associated with combinatorial processing of grammatical morphemes (Gwilliams, 2020; A. Leminen et al., 2016; Marslen-Wilson \& Tyler, 2007; Whiting et al., 2013). The ATL has been found to play a crucial role in semantic memory and conceptual combinatorics, while the posterior temporal lobe, especially the pSTG and pMTG, have been linked to lexico-semantic access (Baron \& Osherson, 2011; Binder et al., 2009; Del Prato \& Pylkkänen, 2014; Friederici, 2012; Gwilliams, 2020; Hickok \& Poeppel, 2007; Turken \& Dronkers, 2011). 
We formulated our predictions based on the previous electromagnetic and electrophysiological studies, which used a passive listening approach to investigate morphosyntactic and morphological processing, typically in an oddball or violation paradigm (e.g., Bakker et al., 2013; Hasting \& Kotz, 2008; Pulvermüller et al., 2008). Contrasting morphologically/morphosyntactically correct forms with ill-formulated forms, these previous studies have found a smaller passive auditory response to morphosyntactically correct combinations of words/morphemes than grammatically incorrect combinations. In grammatical forms, the associative link between frequently co-occurring stems and suffixes may lead to reduced response to the suffix due to preactivation/priming effects elicited by the related stem (Bakker et al., 2013; Hasting \& Kotz, 2008; Pulvermüller et al., 2008). Therefore, in the present study, preactivation effects may play a role in the processing of real word stem + real suffix combinations, while such effects are not expected for novel complex forms that have no pre-existing associative link between the stem and the suffix (e.g., pseudoword stem - "sava" or real word stem "savu" (smoke) + novel suffix - "pe": savape/savupe). Importantly, the ill-formulated forms used in the earlier studies were compiled from existing morphemes in an ungrammatical manner, thus, both the stems and suffixes were familiar in themselves to the participants (e.g., fly+ed in Bakker et al., 2013). The novel complex forms in our study do not violate any morphological rule, but contain novel morphemes, that are initially unfamiliar, but are expected to undergo learning over the course of the experiment. Other studies contrasting novel stem + real suffix combinations (A. Leminen et al., 2013) or real word stem + novel suffix combinations (Kimppa et al., 2019) with familiar complex words reported a stronger activation for familiar stimuli, possibly due to the activation of long-term memory traces. However, none of these studies focused on comparing online learning effects emerging during the perceptual exposure. Therefore to predict possible online changes elicited by the different word types, we also rely on the results of rapid word learning studies that have repeatedly associated memory trace formation with activity enhancement (Kimppa et al., 2015; Shtyrov, 2011; Shtyrov et al., 2010; Yue et al., 2014).

Based on previous findings on learning of suffixes with a defined meaning (A. Leminen et al., 2016; Tamminen et al., 2012, 2015), we hypothesized that novel semantically trained suffixes combined with real word stems will elicit stronger source activations particularly in the STG, MTG and ATL than untrained suffixes, because of initial episodic memories acquired through the semantic training. Real suffixes are expected to be morphologically decomposed in the early stages of processing and so are novel complex words with semantically trained suffixes, reflected in the increased LIFG activity (A. Leminen et al., 2016). We predicted that the repetitive presentation following semantic training will further facilitate memory trace build-up (Kimppa et al., 2015; Shtyrov, 2011; Shtyrov et al., 2010; Yue et al., 2014), hence, we expected no differences in the neural activity to trained novel suffixes and real suffixes at least by the end of passive listening. If rapid perceptual learning is also possible for suffixes, similarly to novel monomorphemic words, then we expect these learning effects to manifest in an activation enhancement also for untrained suffixes by the end of the passive exposure. Pseudoword stems were included as controls, to be able to track the influence of stem lexicality on decomposition. We expected that the lexicality of the stem would affect the processing of suffixes, as it has been suggested that a familiar stem is needed to initiate morphological decomposition (Lehtonen et al., 2007; Leinonen et al., 2009; Prins et al., 2019). Consequently, in the initial phase of the passive listening session, all pseudoword stem + novel suffix combinations might be treated as monomorphemic pseudowords, reflected in the smaller neural source activity to such combination as compared to other stimulus types. Hence, in the absence of a familiar stem, similar learning effects as those expected in case of real word stems should not occur.

\section{Materials and Methods}

\subsection{Participants}

Nineteen healthy, native Finnish speakers participated in the study ( 13 females; mean age $=26$ years, $\mathrm{SD}=4.34$; range: $20-34$ years). All participants had normal or corrected-to-normal vision; none reported any hearing deficits, or psychiatric/neurological conditions. All participants were right-handed, which was verified by the Edinburgh Handedness Inventory (Oldfield, 1971). Ethical permission for 
the study was issued by the Research Ethics Committee of the Helsinki and Uusimaa Hospital District and the procedures conformed to the Declaration of Helsinki. All individuals provided written informed consent and received vouchers as a compensation for their participation.

\subsection{Stimuli}

Four novel suffixes were used in the experiment, grouped into two pairs, out of which only one pair was presented in the semantic training session (counterbalanced across participants). The other pair only appeared in the subsequent MEG session. During the semantic training task, both novel suffixes were combined with 20 different familiar word stems and pictures. Crucially, the word stems presented in this task were not used in the MEG session. To investigate the learning of novel derivational suffix without confounding this with any pre-existing morphological representation, we chose two categories for the intended meaning of the suffixes that are not present in the Finnish derivational system: "big" and "artificial" (rotated across the subjects in a counterbalanced fashion).

For the MEG session, we created spoken stimuli with precisely controlled acoustic and phonetic features while manipulating their lexical and morphological status. We used consonant-vowel (CV) syllables and combined them to form six types of trisyllabic (CVCVCV) stimuli: (1) real word stem with real suffix, (2) real word stem with trained novel suffix, (3) real word stem with untrained novel suffix, (4) pseudoword stem with real suffix (5) pseudoword stem with trained novel suffix, and (6) pseudoword stem with untrained novel suffix. In order to create acoustically and phonologically balanced word stems, the 4 pseudoword stems were created by recombining the syllables of the 4 real word stems (see Fig. 1. A). The real stems were savu (smoke), laji (species; kind/sort/type), tuma (nucleus), and kuva (picture). The pseudoword stems were sava, lavu, tuji, and kuma. The real suffixes category contained 2 items: the interrogative suffix $-k o$, and the derivational suffix $-k e$, referring to either diminutive forms or forms resembling the meaning of the stem noun, were savuke (cigarette), lajike (variety/strain), tumake (nucleus of the central nervous system), and kuvake (icon). Both novel suffix category (trained and untrained novel suffixes) included 2 items, $-p e$ and $-t u$ in one, and $-k u$ and $-t i$ in the other. The role of a certain novel suffix pair was counterbalanced across participants: different novel suffixes were trained for different subjects. Each real word and pseudoword stem was presented in combination with all trained and untrained as well as real suffixes, resulting in 8 unique tokens for each stimulus type, which were presented an equal number of times. Therefore, the type of the attached suffix could not be predicted based on the stem, but only at the suffix onset, i.e., the third syllable; hence, the recognition points were controlled. We ruled out possible effects of individual suffix specific properties on the group-level MEG patterns by cross-splicing stems and suffixes and counterbalancing suffixes across conditions and participants.

All stimuli were produced from digital recordings $(44.1 \mathrm{kHz}, 16 \mathrm{bit})$ of a female native speaker of Finnish. The speaker uttered the first syllables in isolation, while the second and third syllables were uttered in combination with a preceding vowel to make them unstressed, in accordance with the Finnish phonology. The syllables that best matched in their fundamental frequency (F0) and vowel duration were selected from a recording of multiple utterances. We normalized the original syllables by the maximal peak amplitude and matched their durations to $140 \mathrm{msec}$ with $5 \mathrm{msec}$ fade-in and $20 \mathrm{msec}$ fade-out envelopes. The F0 contours of the vowels were adjusted to match the contours of equivalent short Finnish vowels. We equalized the F0 and loudness across syllables, then decreased the F0 of the final syllables by three semitones $(\sim 38 \mathrm{~Hz})$ and loudness by $2 \mathrm{~dB}$ to conform to the natural stress and prosody of spoken Finnish (Suomi et al., 2003). The final tokens were formed by cross-splicing the first, second and third syllables together with a $40 \mathrm{msec}$ silent closure in-between them, typical of Finnish CV onsets. The aim was to meticulously control the acoustic features of syllables to allow precise definition of the divergence point after which both the lexicality of the stem (real or pseudoword) and the morphosyntactic status of the suffix (real, trained or untrained novel suffix) could be identified. The stimulus production and preparation was carried out using Pro Tools 9 (Avid Technology, Inc., Burlington, MA, USA), Adobe Audition 3.0 (Adobe Systems Inc., San Jose, CA, USA) and Praat 5.1.45 (Boersma \& Weenink, 2009).

\subsection{Procedure}




\subsubsection{Training of suffix semantics}

The experiment started with a semantic training session. The task was intended to provide information about the meaning of the presented novel suffixes for the participants. During each trial, a real word appeared on the left side of the screen, while the same word stem combined with a novel suffix appeared on the right. Both words were presented along with pictures depicting their meaning: the image above the familiar word presented a commonly recognizable version of the object, while the image above the novel suffixed word depicted the same object with a salient added attribution corresponding to the meaning carried by the novel suffix. Upon each trial, both the stem and the suffixed word were also auditorily presented (Fig. 1. B \& C). Participants were instructed to pay careful attention to both the words and corresponding pictures and try to understand their meaning. Stimulus presentation was selfpaced using a button press. After completing the task, the participants had to describe the meaning of the trained novel suffixes. All but one participant completed this task successfully after one presentation round; one participant performed the training phase twice to discover the meanings.

\subsubsection{Passive listening}

The semantic training was immediately followed by an MEG recording involving a passive listening task. During the task, the spoken stimuli were presented binaurally through plastic tubes with non-magnetic earpieces at a comfortable sound intensity level. The stimuli consisted of suffixed words including 4 real and 4 pseudoword stems combined with 2 real and 4 novel suffixes. Two of the novel suffixes were presented previously during the semantic training and two were totally novel, with no given meaning. Crucially, the word stems presented in this task were not used in the MEG session pretrained suffixes were attached to other stems, to investigate specifically the suffix (rather than wholeform) learning. Each stimulus type was repeated 200 times ( 25 repetition per token) during the exposure session in a pseudo-random sequence to ensure a balanced occurrence of each token throughout the experiment. The average stimulus onset asynchrony (SOA) was $900 \mathrm{msec}$ with a $\pm 10-50 \mathrm{msec}$ jitter in 10-msec steps. During the stimulus presentation, the participants were asked to focus on watching a silent animated videofilm without subtitles and ignore the spoken input, as they were required to complete a 13-item questionnaire on the movie content after the experiment. To reduce fatigue, the recording was broken into three 6-minute-long blocks with short breaks in-between (Fig. 1. B). 
A)

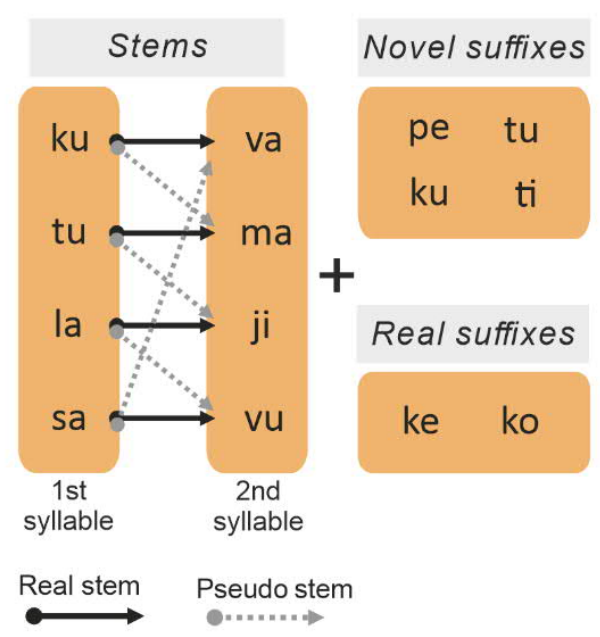

B)
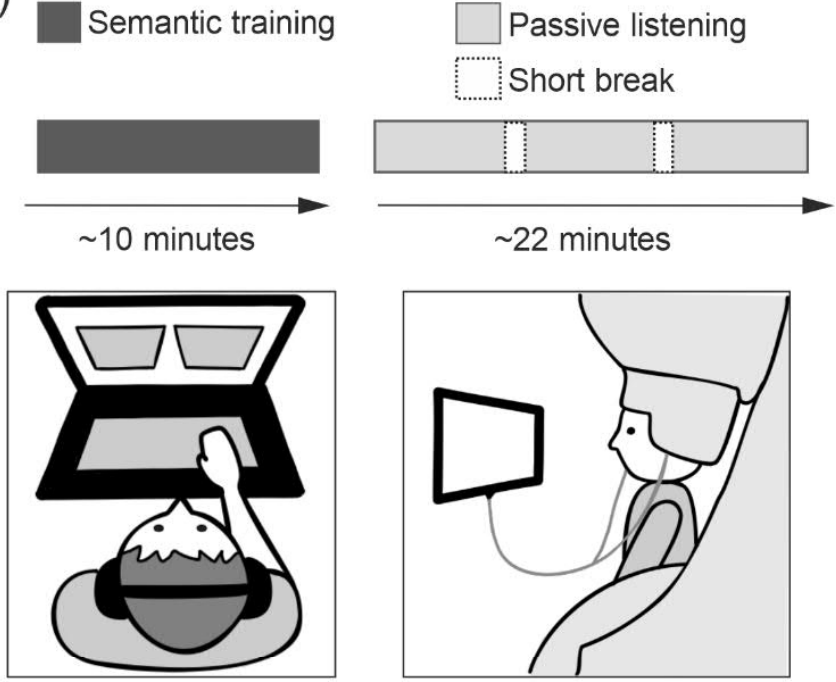

C)

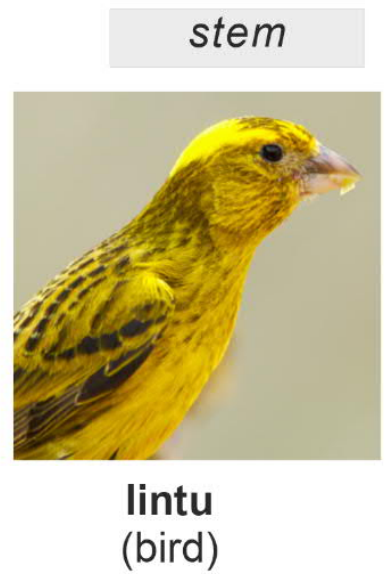

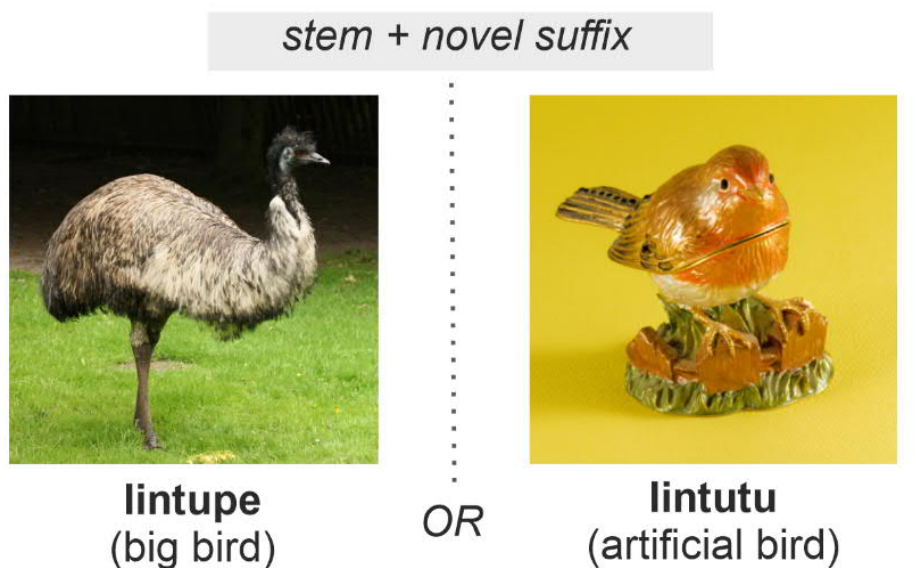

(artificial bird)

Fig. 1. - (A) Stimuli used in the passive listening task consisted of matched sets of meaningful real word stems (savu - smoke, laji - species, tuma - nucleus, kuva - picture) and novel phonotactically and phonologically legal pseudoword stems (sava, lavu, tuji, kuma), each item combined with a real suffix (ke - diminutive suffix, ko question clitic) or a trained or untrained novel suffix (-pe, -tu, -ku, -ti). (B) Schematic presentation of the experimental design. The short semantic training was followed by the passive listening task, during which continuous MEG was recorded. (C) Examples of the novel suffixes combined with real word stems and their meanings, illustrated by pictures. The semantic training task included two different novel suffixes (e.g. - pe, - tu) and the remaining two suffixes were used as untrained novel suffixes (e.g. $-\mathrm{ku},-\mathrm{ti}$ ) later, in the passive listening task only. The real word stems used in this task, such as 'lintu' (bird), were not presented during the passive listening task. The use of either pair of suffixes was counterbalanced across trained versus untrained stimuli and participants.

\subsection{Data acquisition and analysis}

The recordings were carried out in a magnetically shielded room using a Vectorview ${ }^{\mathrm{TM}}$ whole head MEG system (Elekta Neuromag, Elekta Ltd., Helsinki, Finland). The system contains 204 orthogonal planar gradiometers and 102 magnetometers, resulting in 306 data channels. Eyemovements and blinks were monitored by horizontal electro-oculogram (HEOG) using a pair of bipolar electrodes placed at the temples and vertical EOG with two electrodes attached above and below the left eye. To determine the head position inside the recording device, locations of three head position indicator (HPI) coils were recorded in relation to both the cardinal points of the head (nasion, left and right preaurical points, which were identified prior to the experiment with an Isotrak 3D-digitiser (Polhemus, Colchester, VT, USA) and the MEG sensors in the device helmet. The sampling rate was $600 \mathrm{~Hz}$ and the MEG signals were band-pass filtered online at 0.1-200 Hz. 
Raw MEG data were first pre-processed with the spatiotemporal signal space separation (tSSS) algorithm (MaxFilter software, Elekta Neuromag) to correct for artefacts produced by external interference (e.g., line noise) and other nearby sources (e.g., the heart; Taulu \& Simola, 2006). Further analyses were carried out using the Brainstorm toolbox for MATLAB (MathWorks, Natick, MA; Tadel et al., 2011). The continuous data were low-pass filtered with $40 \mathrm{~Hz}$ corner frequency (39-point twoway least-squares FIR filter with $7 \mathrm{~Hz}$ transition band). Signal space projection (SSP) algorithm was used to correct for artefacts caused by eye-blinks (Tesche et al., 1995). Epochs time-locked to the suffix onset, with $-40 \mathrm{~ms}$ pre- and $500 \mathrm{~ms}$ post-suffix intervals were extracted and baseline corrected using interval from $-40 \mathrm{~ms}$ to $0 \mathrm{msec}$ (corresponding to the silent closure between the second and the third syllables, determining the relatively short baseline duration). Epochs containing baseline fluctuations, muscle and horizontal eye movement-related artefacts were removed using $3000 \mathrm{fT} / \mathrm{cm}, 6000 \mathrm{fT}$ and $\pm 150 \mu \mathrm{V}$ rejection thresholds for gradiometers, magnetometers and EOG channels, respectively.

Our aim was to investigate the neural signatures of the rapid acquisition of the presented novel suffixes by comparing neural activity early and late in the exposure. To this end, similarly to previous studies investigating rapid learning, we created averages of the first and last $25 \%$ of the trials to account for the early and late stages of exposure to each word type (Kimppa et al., 2015; Shtyrov, 2011; Shtyrov et al., 2010). On average, the number of trials for the late and early responses was $40.1(\mathrm{SD}=6.4)$ per condition. To determine the cortical generators of the learning-related effects, we performed distributed source analysis. The forward solution was created using overlapping spheres with full noise covariance matrix. We used L2 minimum norm estimation (MNE) to assess the neuronal current density maps underlying the measured gradiometer and magnetometer data. Source reconstruction was performed for each participant, stimulus category and time point. Normalization using Z-score transformation and spatial smoothing was carried out on the source maps and they were projected onto the standardized Montreal Neurological Institute (MNI) brain.

\subsection{Statistical analysis}

For unbiased estimation of activity, the time windows of interest were centered around peaks identified in the global field power (GFP) waveform averaged across all participants and experimental conditions, which were located at $130 \mathrm{~ms}$ and $240 \mathrm{~ms}$ (Fig. 2). Respectively, to analyze source strength, we computed area mean source amplitudes for all individual stimulus types at the two time windows: 120-140 ms, and 220-260 ms after the suffix onset. The second time window was set to be wider than the first (40 ms as compared to $20 \mathrm{~ms}$ ), because the later effect appeared to last longer than the earlier one (Fig. 2). We investigated four regions of interest in the left hemisphere, which were defined a priori, based on previous literature on neural language processing (see Introduction): IFG, ATL, posterior STG and posterior MTG (Binder et al., 2009; Bozic \& Marslen-Wilson, 2010; Friederici, 2012; Gwilliams, 2020). The ROIs were defined on the standardized MNI template using the Desikan-Killiany cortical atlas (Desikan et al., 2006).

To assess the activation dynamics for the different suffix types over the course of the exposure, we conducted repeated measures analysis of variance (ANOVA) implemented in SPSS Statistics v. 25 software (IBM, Armonk, NY) for the two time windows with the following within-subject factors: Stem lexicality (2 levels: real word, pseudoword) $\times$ Suffix (3 levels: trained novel suffix, untrained novel suffix, real suffix) $\times$ Exposure time (2 levels: early vs. late in exposure) $\times$ ROI (IFG, ATL, posterior STG, posterior MTG). In the additional analysis that was preformed to assess the strength of the memory traces of the novel suffixes, by comparing the early responses to real suffixes with the late responses to novel suffixes, the effect of Exposure time was not included. Significant interactions were followed up using Bonferroni-corrected pairwise comparisons. Significance level was set to .05; the $\eta_{p}^{2}$ effect size is reported for each significant effect. Greenhouse-Geisser-corrected F and p values are reported if the assumption of sphericity was violated based on Mauchly's Test. All significant effects are reported. 


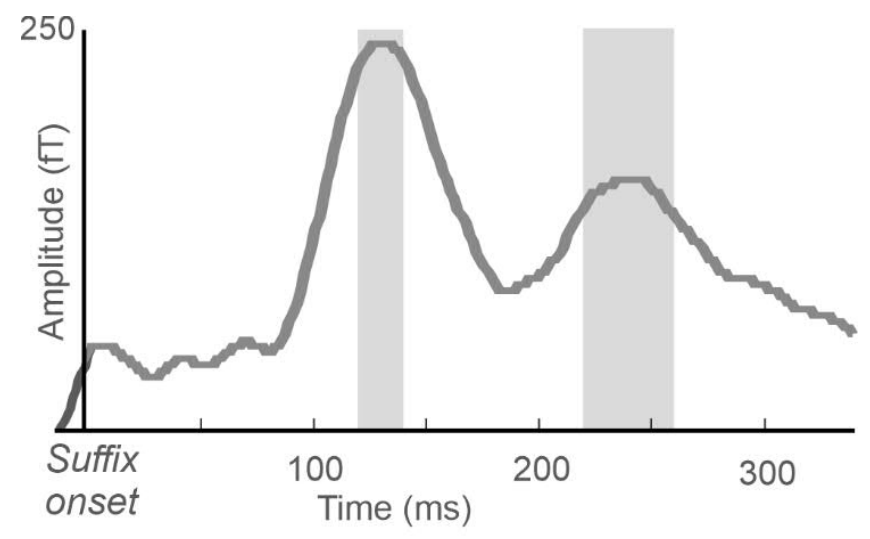

Fig. 2. Global field power of the event-related field time-locked to suffix onset over all stimulus types. For source reconstruction, time windows around $130 \mathrm{~ms}$ and $240 \mathrm{~ms}$ were chosen based on the most prominent peaks.

\section{Results}

\subsection{Task compliance}

The hit rate in the questionnaire about the videofilm content was high, with a mean of $99.34 \%$ correct $(\mathrm{SD}=2.79)$ from the 13 questions. This result confirms that participants complied with the primary task of paying attention to the video during the MEG recording.

\subsection{MEG results}

\subsubsection{Comparing the activation dynamics of the different suffix types}

In the 120-140-ms time window, there was a significant main effect of $\operatorname{Suffix}(F(1.31,23.66)=$ $\left.13.815, p<.001, \eta_{p}^{2}=.434\right)$; post-hoc tests indicated that regardless of the lexicality of the stem and phase of presentation, real suffixes elicited significantly higher activation than either trained $(p=.003)$ or untrained $(p=.003)$ novel suffixes. We also found that the activation patterns differ across the ROIs $\left(F(3,54)=17.360, p<.001, \eta_{p}^{2}=.434\right)$, which was due to pMTG activity being significantly higher than IFG $(p<.001)$, ATL $(p=.009)$ and pSTG $(p=.001)$ activity. ATL activity was also higher than that in the pSTG $(p=.036)$. The Suffix $x$ ROI interaction $\left(F(2.06,37.03)=9.770, p<.001, \eta_{p}^{2}=.352\right)$ revealed that the increased source activity elicited by real suffixes compared with trained $(p<.001)$ and untrained $(p=0.001)$ novel suffixes was only present in the pMTG. There was also a significant interaction of Stem x Early/Late exposure $\left(F(1,18)=9.923, p=.006, \eta_{p}^{2}=.355\right)$. Based on post-hoc tests, no difference was found between suffixes with real word and pseudoword stems early in exposure $(p=.159)$. However, suffixes combined with pseudoword stems showed a significant decrease ( $p=$ .021 ), while suffixes with real word stems showed a marginal increase of neural activity over the course of the experiment ( $p=.059)$ (Fig. 3. A \& B). Furthermore, the Stem $x$ Suffix $x$ Early/Late exposure interaction $\left(F(2,36)=3.800, p=.032, \eta_{p}^{2}=.174\right)$ revealed that the aforementioned decrease in activity to suffixes with pseudoword stems was most prominently observable for real and untrained novel suffixes ( $p=.030, p=.035$, respectively), while it was not significant for trained novel suffixes $(p=$ .676). There was a marginally significant activity increase between early and late responses, elicited by untrained novel suffixes paired with real word stems $(p=.078)$ (Fig.3.A \& B).

In the 220-260 ms time window, we found a significant Suffix main effect $(F(1.18,21.16)=$ $\left.13.815, p=.039, \eta_{p}^{2}=.203\right)$; real suffixes evoked marginally higher source activation than untrained (but not trained) novel suffixes $(p=0.098)$ (Fig. 3. A). Similarly to the earlier time window, source amplitudes differed across the ROIs $\left(F(1.99,30.99)=5.692, p=.007, \eta_{p}^{2}=.240\right)$ : pMTG and ATL showed the strongest activation, both were significantly higher compared to pSTG ( $p=.048, p=.001$, respectively). 
B)

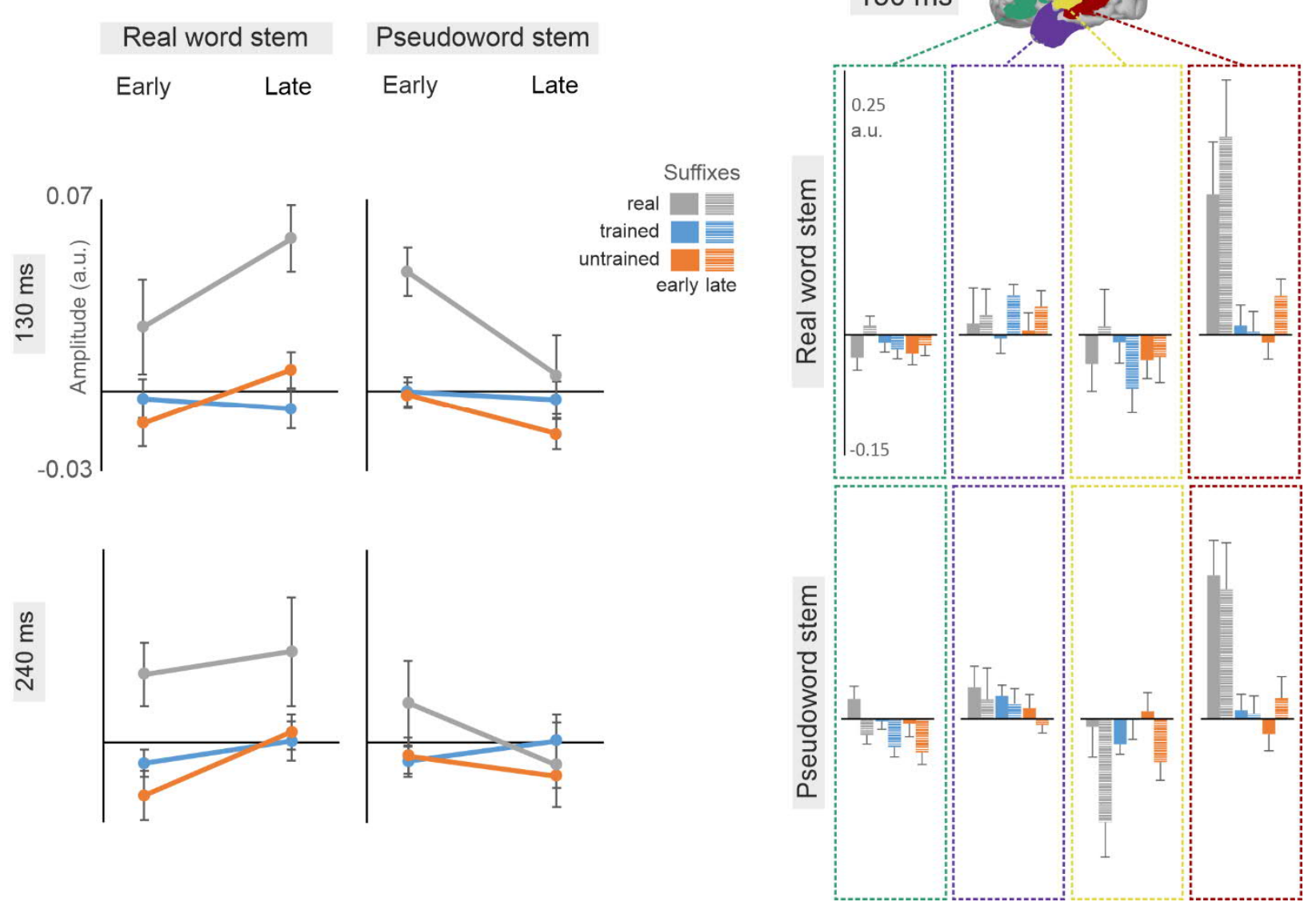

Fig. 3. (A) Average source activations across all ROIs early and late in exposure time at $130 \mathrm{~ms}$ and $240 \mathrm{~ms}$ after suffix onset, separately for the different stems and suffixes, collapsed across regions of interest. At $130 \mathrm{~ms}$ untrained suffixes paired with real word stems show a marginal increase. However, both real and untrained novel suffixes elicited a significant decrease over the exposure time when paired with pseudoword stems. In the late time window, real suffixes elicit higher activation than untrained (but not trained) novel suffixes, regardless of the exposure time and lexicality of the stem. Higher activation is also observed when they are combined with real word stems compared to pseudoword stems. Overall source activation for existing complex forms, i.e., real suffixes paired with real word stems, is stronger than for novel suffixes. (B) Source activation at $130 \mathrm{~ms}$ in the early and late phases of passive exposure in each ROI. The observed activation patterns are strongly influenced by the stem lexicality: in general, pseudoword stems contribute to a response decrease, while real word stems to a response increase over the exposure time. Activation is shown in arbitrary units (a.u.) based on the z-score normalization of the current density maps. The error bars mark the standard error.

\subsubsection{Comparing early responses to real suffixes vs. late responses to novel suffixes}

In the 120-140 ms time window, we found a significant main effect of $\operatorname{Suffix}(F(1.18,21.17)=$ $\left.9.418, p=.004, \eta_{p}^{2}=.343\right)$; post-hoc tests indicated that, irrespective of stem lexicality, real suffixes elicited stronger source activations than novel trained $(p=.009)$ and untrained $(p=.026)$ suffixes. Additionally, we found a difference in activation strength across the $\operatorname{ROIS}(F(3,54)=13.921, p<.001$, $\left.\eta_{p}^{2}=.436\right)$ : pMTG activity was significantly higher than IFG $(p<.001)$, and pSTG $(p=.001)$ activity. The Suffix $x$ ROI interaction $\left(F(2.45,44.14)=4.321, p=.014, \eta_{p}^{2}=.194\right)$ indicated that the increased source activity elicited by real suffixes compared with trained $(p=.004)$ and untrained $(p=.042)$ novel suffixes was only present in the pMTG. Interestingly, the significant Stem $x$ Suffix interaction $(F(2,36)$ $\left.=3.350, p=.046, \eta_{p}^{2}=.157\right)$ revealed that differences between real and novel trained $(p=.003)$ and untrained $(p<.001)$ suffixes only emerged in case of pseudoword stems, while the activation was comparable for all suffix types combined with real word stems $(p>.269)$ (Fig. 4). 
In the 220-260 ms time window, the main effect of ROI $\left(F(3,54)=5.252, p=.003, \eta_{p}^{2}=.226\right)$ indicated that source activations differed across ROIs and were generally highest in the ATL, this effect being significant in comparison between ATL and pSTG $(p=.001)$. We also found a difference between the suffix types (the main effect of Suffix: $F(2,36)=4.559, p=.017, \eta_{p}^{2}=.202$ ), such that real suffixes evoked significantly higher source activation than untrained (but not trained) novel suffixes $(p=.027)$.

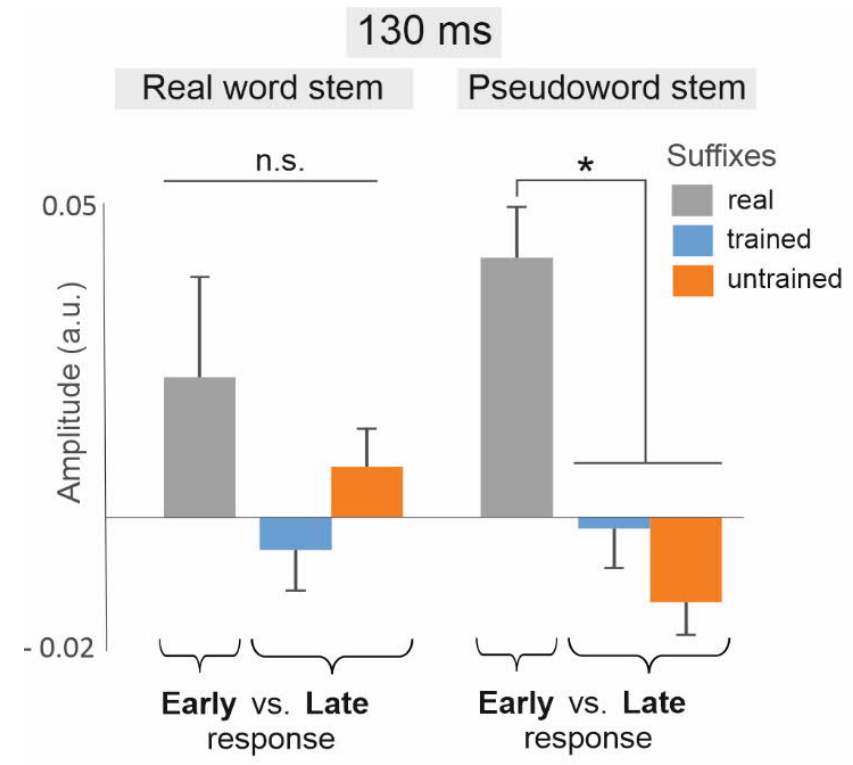

Fig. 4. Source activation to real suffixes early in exposure and to novel trained and untrained suffixes late in exposure in the early time window, collapsed across regions of interest. Significant differences between real and novel trained and untrained suffixes only emerge in case of pseudoword stems, but not real word stems. Activation is shown in arbitrary units (a.u.) based on the z-score normalization of the current density maps. The error bars mark the standard error.

\section{Discussion}

In the present study, we 1) investigated neural changes underlying the online acquisition of previously unfamiliar suffixes in the context of familiar real word and unfamiliar pseudoword stems during a passive auditory exposure and (2) examined whether this automatic learning process benefits from/is speeded up by semantic information associated with the novel suffixes modulates. We will first briefly summarize our main results and then discuss each result in detail.

We found that around $130 \mathrm{~ms}$ post-suffix onset - differences emerged in the neural source activation elicited by the different suffixes: in the posterior MTG, we found a significantly stronger source activation to real suffixes than to both types of novel suffixes. This effect was present irrespective of the lexicality of the stem and did not change over the time course of the passive exposure. We also found a divergent pattern of changes in source activation between the early and late phases of exposure depending on the lexicality of the stem: activation to real and untrained suffixes combined with pseudoword stems significantly decreased, while untrained suffixes combined with real word stems marginally increased over time. In the later time window, at around $240 \mathrm{~ms}$ post suffix, real suffixes elicited stronger activation than untrained novel suffixes, which was not modulated by the stem or the exposure time, while no differences were observed between the real and trained novel suffixes. Additionally, when comparing the early responses to real suffixes with late responses to novel suffixes, in the 120-140 ms time window, we found that source activation elicited by either type of novel suffix did not differ from real suffixes when they were attached to real-word stems, whereas when combined with pseudoword stems, real suffixes evoked higher activation than both novel suffix types. In the 220260 time window, we found a higher overall activation for real suffixes than untrained novel suffixes, while no differences were observed between real suffixes and trained novel suffixes.

One of the main goals of our study was to examine whether semantics benefit suffix learning. We expected that semantic information will facilitate the learning process, manifesting as an activity enhancement, but this might not be possible in case of purely perceptual learning. We indeed observed 
differences between the processing of semantically trained and untrained novel suffixes at around 130 ms after the suffix onset. However, despite our predictions, semantically trained novel suffixes showed a consistent activation pattern throughout the passive listening task, irrespective of the stem type. This result is in line with previous behavioral findings about immediate learning effects for novel derivational suffixes (Merkx et al., 2011; Tamminen et al., 2015). Tamminen and colleagues (2015) suggested that initial episodic memories acquired during semantic learning may elicit immediate learning effects (which, in their case, was expressed in non-speeded tasks), but further lexicalization can only be achieved over a longer period of time. Moreover, Merkx and colleagues (2011), observed that semantics have a positive immediate effect on suffix learning in the context of familiar stems, albeit these effects generalize to new stems after an offline consolidation stage. Hence, the lack of significant activity enhancement for trained novel suffixes during the passive exposure, in our experiment, could potentially be the result of a ceiling effect due to the initial training that precluded further facilitation through repetitive passive exposure. It is likely that twenty presentations during the semantic training task, followed by an explicit report provided by the participants about the meaning of the novel suffixes, were enough for immediate memory-trace formation, but further repetitions did not seem to have an additional learning effect. By contrast, importantly, we found that untrained novel suffixes elicited an activation pattern modulated by the lexicality of the stem that was similar in direction but significantly lower in amplitude than real suffixes. We observed an activity enhancement for untrained novel suffix + real word stem combinations from the early to the late phase of the passive exposure. Since untrained suffixes were encountered for the first time during the passive exposure, they could only undergo perceptual learning, yet we see clear evidence for learning and, most likely, morphological decomposition. As indicated by several studies investigating the rapid learning of simple words in a passive manner, without attention, this enhancement is likely indicating memory-trace formation (Shtyrov, 2011; Shtyrov et al., 2010; Yue et al., 2014). Taken together, the distinct activity patterns for trained and untrained items indicate that semantic information is an important factor modulating the suffix learning process and giving a head start for morphological sensitivity, promoting almost immediate morphological decomposition.

The role of the real suffixes in our paradigm was to serve as a control measure, to better understand and track the learning process and possible morphological decomposition effects of the novel items, particularly semantically trained ones. These familiar suffixes were presented in the context of both real and pseudoword stems, which - as expected - did modulate their observed source activation dynamics over the course of the repetitive exposure; this stem lexicality effect was seen in the 120-140 ms time window. Our results showed that the activation for real suffixes appeared to be increasing, although not significantly, by the late phase of the passive exposure, when they were combined with real words. This finding may seem contradictory to previous results suggesting that familiar stems in grammatically correct combinations may elicit a priming effect on the suffix, potentially leading to reduced activation (Bakker et al., 2013; Hasting \& Kotz, 2008; Pulvermüller et al., 2008). Importantly, however, here we combined these suffixes with several different stems during the task; these complex forms undergoing morphological decomposition may have resulted in the various familiar combinations gradually activating widespread cortical networks over time (Kimppa et al., 2015; Shtyrov, 2011; Shtyrov et al., 2010). Conversely, the decreasing activation to real suffixes paired with pseudoword stems may indicate that decomposition is hindered in case the stem is unfamiliar, as it might be much more difficult to distinguish the morphemes constructing the complex words. This corroborates previous findings with active reading and listening paradigms, proposing that morphological decomposition requires a recognizable stem (Lehtonen et al., 2007; Leinonen et al., 2009; Prins et al., 2019).

Overall, real suffixes showed a higher activation than novel suffixes in the early phase and continued to do so by the late phase, irrespective of whether they showed an increase (paired with real word stems) or decrease (pseudoword stems). With respect to their neuroanatomical underpinnings, we found that these differences emerged in the posterior MTG, a region that has been linked to semantic processing, which might be the reason why real suffixes with the most established semantic references activate this area more than novel ones (Binder et al., 2009; Gwilliams, 2020; Turken \& Dronkers, 2011). However, the online changes in the activation to real and untrained suffixes from early to late exposure detected around $130 \mathrm{~ms}$, were not specific to this one particular region, but the whole network. 
We further assessed the strength of memory traces of real and novel suffixes by comparing the source activation to real suffixes in the early phase (before the abovementioned factors start affecting their processing) with that to the novel suffixes in the late phase of the passive exposure. We expected that the source activation to semantically trained suffixes would not differ from real suffixes, but mere perceptual exposure to novel suffixes may not be sufficient for learning. Interestingly, we found no significant differences in the source activation between familiar and novel suffix types when they were paired with real word stems. This suggests that both semantic and perceptual learning can lead to similar memory trace build-up. Although the activation to trained suffixes did not change between the early and late phases, while the activation evoked by untrained suffixes increased over time, these different activation dynamics of the two types of novel suffixes nevertheless indicate that their learning may rely on distinct processes.

In the late time window, real suffixes again yielded the strongest source activity. However, irrespective of the stem type or the exposure time, they did not differ significantly from trained novel suffixes, and we found only marginally significant differences compared to untrained items. When comparing the activation elicited by novel suffixes in the late phase with real suffixes in the early phase, we found similar effects, although the difference between real and untrained suffixes was statistically significant. The lack of changes between the early and late phases in this time window and lack of differences between real and trained suffixes suggest that this processing phase is involved in semantic and combinatorial processes of combining the meanings of the novel suffix and the stem (Gwilliams, 2020)

Overall, our MEG results indicate that neural signatures of memory trace formation already emerge during a short repetitive passive exposure, but it may not be sufficient in itself for the complete integration of novel suffixes to the mental lexicon, indicated by a higher overall activation to real versus novel suffixes throughout the MEG session. Similar neural source activity between trained and untrained novel suffixes suggests that learning of suffixes takes place during both learning types, i.e., perceptual exposure without focused attention on the stimuli and semantic learning. Untrained suffixes were first encountered during the passive listening task, therefore, the initial steps of memory trace formation for these items are indicated by activation enhancement over the exposure. The unchanging activation of trained suffixes detected during the same task suggests that, after the preliminary explicit learning, these items do not benefit from additional perceptual exposure, and automatic morphological decomposition is initiated already in the beginning of perceptual exposure to the suffixes combined with novel stems. In other words, only 20 repetitions of the novel suffixes are enough for morphological analysis to be initiated even without focused attention on the stimuli. Our findings further suggest, however, that such automatic morphological decomposition is also applied for the untrained, purely perceptually learned novel suffixes, but this parsing process requires significantly more repetitions (200 repetitions in the present study). Since the activation evoked by trained suffixes did not change between the early and late phases, while for untrained suffixes it increased over time, these differences between their activation dynamics imply that perceptual and semantic learning strategies involve different memory processes. Through the initial semantic training task, followed by direct questions about the decoded meanings of the suffixes, the participants gained explicit knowledge about the underlying semantics of the novel items as compared to purely perceptual exposure. While these trained morphemes were then further presented in the passive listening task, because of the additional explicit knowledge, their learning process was essentially different from those that were presented repetitively without focused attention or semantic context. This interpretation would corroborate the study of Ferman and colleagues (2009), who suggested that declarative memory contributes to learning the semantic aspects of a morphological rule, while phonological information is more likely acquired through procedural learning. When studying the time course of a few days of learning, Nevat et al. (2017) concluded that the inflection of untrained words engages brain regions associated with procedural learning, in contrast with trained words. These possibly distinct underlying processes could explain the different activation patterns found for novel suffixes undergoing semantic versus perceptual learning.

\section{Conclusions}


We investigated the neuromagnetic signatures of online suffix learning with an emphasis on the role of semantic information on this process. Our findings demonstrate that semantic training modulates the learning process, indicated by the different response patterns evoked by trained and untrained novel suffixes 130 milliseconds after the suffix onset. Pre-trained suffixes showed a consistent activation pattern throughout the learning task, an effect that possibly reflects the formation of initial episodic memories during the semantic training, giving a head-start for morphological decomposition. By contrast, we found an activation increase for untrained suffixes combined with real word stems. Over the course of the passive auditory exposure reflecting gradual suffix-related memory trace formation through perceptual learning, leading to morphological analysis applied for these suffixes towards the end of the experiment. The differences in the learning dynamics between the two suffix types may also suggest at least partially distinct underlying learning mechanisms: untrained suffixes with purely phonological information may be learned through procedural memory mechanisms, while trained suffixes with more explicit, semantic information are encoded in declarative memory. Furthermore, by contrasting the strength of immediate memory trace activation of real suffixes (early phase) with the result of novel suffix learning (late phase), we found that when combined with real word stems, neural source activations to both types of novel suffixes were comparable with familiar suffixes, reflecting formed memory traces for novel suffixes, triggering morphological analysis for all suffix types. Overall, our findings demonstrate almost immediate morphological parsing effects of suffix acquisition gained through semantic learning and more incremental effects of morphological analysis for suffix acquisition obtained through purely perceptual exposure.

\section{Funding statement}

This study was supported by the University of Helsinki (own research funds to AL, as well as CLIC Doctoral Programme to VB), Academy of Finland (grant 330391 to LK), Lundbeck Foundation (grant R140-2013-12951 to YS, project 15480 Neolex); Danish Council for Independent Research (grant DFF 6110-00486 to YS, project 23776).

\section{Author Contributions Statement}

AL, LK, ML, VB, and YS contributed to conception and design of the study. VB performed the data analyses and wrote the first draft of the manuscript. AL, LK, ML, and YS wrote sections of the manuscript. All authors contributed to manuscript revision, read, and approved the submitted version.

\section{Declaration of interest}

The authors have no financial or personal conflicts of interest.

\section{References}

Amenta, S., \& Crepaldi, D. (2012). Morphological Processing as We Know It: An Analytical Review of Morphological Effects in Visual Word Identification. Frontiers in Psychology, 3. https://doi.org/10.3389/fpsyg.2012.00232

Bakker, I., MacGregor, L. J., Pulvermüller, F., \& Shtyrov, Y. (2013). Past tense in the brain's time: Neurophysiological evidence for dual-route processing of past-tense verbs. NeuroImage, 71, 187-195. https://doi.org/10.1016/j.neuroimage.2012.12.065 
Baron, S. G., \& Osherson, D. (2011). Evidence for conceptual combination in the left anterior temporal lobe. NeuroImage, 55(4), 1847-1852. https://doi.org/10.1016/j.neuroimage.2011.01.066

Binder, J. R., Desai, R. H., Graves, W. W., \& Conant, L. L. (2009). Where Is the Semantic System? A Critical Review and Meta-Analysis of 120 Functional Neuroimaging Studies. Cerebral Cortex, 19(12), 2767-2796. https://doi.org/10.1093/cercor/bhp055

Boersma, P., \& Weenink, D. (2009). Praat: Doing Phonetics by Computer. http://www.fon.hum.uva.nl/praat/

Bozic, M., \& Marslen-Wilson, W. (2010). Neurocognitive Contexts for Morphological Complexity: Dissociating Inflection and Derivation. Language and Linguistics Compass, 4(11), 1063-1073. https://doi.org/10.1111/j.1749-818X.2010.00254.x

Bozic, M., Tyler, L. K., Ives, D. T., Randall, B., \& Marslen-Wilson, W. D. (2010). Bihemispheric foundations for human speech comprehension. Proceedings of the National Academy of Sciences, 107(40), 17439-17444. https://doi.org/10.1073/pnas.1000531107

De Grauwe, S., Lemhöfer, K., Willems, R. M., \& Schriefers, H. (2014). L2 speakers decompose morphologically complex verbs: FMRI evidence from priming of transparent derived verbs. Frontiers in Human Neuroscience, 8, 802. https://doi.org/10.3389/fnhum.2014.00802

Del Prato, P., \& Pylkkänen, L. (2014). MEG evidence for conceptual combination but not numeral quantification in the left anterior temporal lobe during language production. Frontiers in Psychology, 5. https://doi.org/10.3389/fpsyg.2014.00524

Desikan, R. S., Ségonne, F., Fischl, B., Quinn, B. T., Dickerson, B. C., Blacker, D., Buckner, R. L., Dale, A. M., Maguire, R. P., Hyman, B. T., Albert, M. S., \& Killiany, R. J. (2006). An automated labeling system for subdividing the human cerebral cortex on MRI scans into gyral based regions of interest. NeuroImage, 31(3), 968-980. https://doi.org/10.1016/j.neuroimage.2006.01.021

Endress, A. D., \& Hauser, M. D. (2011). The influence of type and token frequency on the acquisition of affixation patterns: Implications for language processing. Journal of Experimental Psychology: Learning, Memory, and Cognition, 37(1), 77-95. https://doi.org/10.1037/a0020210

Ferman, S., \& Karni, A. (2010). No Childhood Advantage in the Acquisition of Skill in Using an Artificial Language Rule. PLOS ONE, 5(10), e13648. https://doi.org/10.1371/journal.pone.0013648

Ferman, S., Olshtain, E., Schechtman, E., \& Karni, A. (2009). The acquisition of a linguistic skill by adults: Procedural and declarative memory interact in the learning of an artificial morphological rule. Journal of Neurolinguistics, 22(4), 384-412. https://doi.org/10.1016/j.jneuroling.2008.12.002

Friederici, A. D. (2012). The cortical language circuit: From auditory perception to sentence comprehension. Trends in Cognitive Sciences, 16(5), 262-268. https://doi.org/10.1016/j.tics.2012.04.001

Gwilliams, L. (2020). How the brain composes morphemes into meaning. Philosophical Transactions of the Royal Society B: Biological Sciences, 375(1791), 20190311. https://doi.org/10.1098/rstb.2019.0311

Hasting, A. S., \& Kotz, S. A. (2008). Speeding Up Syntax: On the Relative Timing and Automaticity of Local Phrase Structure and Morphosyntactic Processing as Reflected in Event-related Brain Potentials. Journal of Cognitive Neuroscience, 20(7), 1207-1219. https://doi.org/10.1162/jocn.2008.20083

Havas, V., Laine, M., \& Rodríguez Fornells, A. (2017). Brain signatures of early lexical and morphological learning of a new language. Neuropsychologia, 101, 47-56. https://doi.org/10.1016/j.neuropsychologia.2017.04.005

Havas, V., Waris, O., Vaquero, L., Rodríguez-Fornells, A., \& Laine, M. (2015). Morphological learning in a novel language: A cross-language comparison: Quarterly Journal of Experimental Psychology. https://journals.sagepub.com/doi/10.1080/17470218.2014.983531

Hickok, G., \& Poeppel, D. (2007). The cortical organization of speech processing. Nature Reviews Neuroscience, 8(5), 393-402. https://doi.org/10.1038/nrn2113 
Kimppa, L., Kujala, T., Leminen, A., Vainio, M., \& Shtyrov, Y. (2015). Rapid and automatic speechspecific learning mechanism in human neocortex. Neurolmage, 118, 282-291. https://doi.org/10.1016/j.neuroimage.2015.05.098

Kimppa, L., Shtyrov, Y., Hut, S. C. A., Hedlund, L., Leminen, M., \& Leminen, A. (2019). Acquisition of L2 morphology by adult language learners. Cortex, 116, 74-90. https://doi.org/10.1016/j.cortex.2019.01.012

Lehtonen, M., Cunillera, T., Rodríguez-Fornells, A., Hultén, A., Tuomainen, J., \& Laine, M. (2007). Recognition of morphologically complex words in Finnish: Evidence from event-related potentials. Brain Research, 1148, 123-137. https://doi.org/10.1016/j.brainres.2007.02.026

Leinonen, A., Grönholm-Nyman, P., Järvenpää, M., Söderholm, C., Lappi, O., Laine, M., \& Krause, C. M. (2009). Neurocognitive processing of auditorily and visually presented inflected words and pseudowords: Evidence from a morphologically rich language. Brain Research, 1275, 54-66. https://doi.org/10.1016/j.brainres.2009.03.057

Leminen, A., Kimppa, L., Leminen, M. M., Lehtonen, M., Mäkelä, J. P., \& Shtyrov, Y. (2016). Acquisition and consolidation of novel morphology in human neocortex: A neuromagnetic study. Cortex; a Journal Devoted to the Study of the Nervous System and Behavior, 83, 1-16. https://doi.org/10.1016/j.cortex.2016.06.020

Leminen, A., Leminen, M., Kujala, T., \& Shtyrov, Y. (2013). Neural dynamics of inflectional and derivational morphology processing in the human brain. Cortex, 49(10), 2758-2771. https://doi.org/10.1016/j.cortex.2013.08.007

Leminen, M., Leminen, A., Smolander, S., Arkkila, E., Shtyrov, Y., Laasonen, M., \& Kujala, T. (2020). Quick reorganization of memory traces for morphologically complex words in young children. Neuropsychologia, 138, 107309. https://doi.org/10.1016/j.neuropsychologia.2019.107309

Marslen-Wilson, W. D. (1985). Speech shadowing and speech comprehension. Speech Communication, 4(1), 55-73. https://doi.org/10.1016/0167-6393(85)90036-6

Marslen-Wilson, W. D., \& Tyler, L. K. (2007). Morphology, language and the brain: The decompositional substrate for language comprehension. Philosophical Transactions of the Royal Society B: Biological Sciences, 362(1481), 823-836. https://doi.org/10.1098/rstb.2007.2091

Merkx, M., Rastle, K., \& Davis, M. H. (2011). The acquisition of morphological knowledge investigated through artificial language learning. Quarterly Journal of Experimental Psychology, 64(6), 1200-1220. https://doi.org/10.1080/17470218.2010.538211

Mirković, J., \& Gaskell, M. G. (2016). Does Sleep Improve Your Grammar? Preferential Consolidation of Arbitrary Components of New Linguistic Knowledge. PloS One, 11(4), e0152489. https://doi.org/10.1371/journal.pone.0152489

Mirković, J., Vinals, L., \& Gaskell, M. G. (2019). The role of complementary learning systems in learning and consolidation in a quasi-regular domain. Cortex, 116, 228-249. https://doi.org/10.1016/j.cortex.2018.07.015

Nevat, M., Ullman, M. T., Eviatar, Z., \& Bitan, T. (2017). The neural bases of the learning and generalization of morphological inflection. Neuropsychologia, 98, 139-155. https://doi.org/10.1016/j.neuropsychologia.2016.08.026

Prins, T., Dijkstra, T., \& Koeneman, O. (2019). How Dutch and Turkish-Dutch readers process morphologically complex words: An ERP study. Journal of Neurolinguistics, 50, 37-52. https://doi.org/10.1016/j.jneuroling.2017.12.003

Pulvermüller, F., Shtyrov, Y., Hasting, A. S., \& Carlyon, R. P. (2008). Syntax as a reflex: Neurophysiological evidence for early automaticity of grammatical processing. Brain and Language, 104(3), 244-253. https://doi.org/10.1016/j.bandl.2007.05.002

Shtyrov, Y. (2011). Fast Mapping of Novel Word Forms Traced Neurophysiologically. Frontiers in Psychology, 2. https://doi.org/10.3389/fpsyg.2011.00340

Shtyrov, Y., Nikulin, V. V., \& Pulvermüller, F. (2010). Rapid Cortical Plasticity Underlying Novel Word Learning. Journal of Neuroscience, 30(50), 16864-16867. https://doi.org/10.1523/JNEUROSCI.1376-10.2010

Steinhauer, K. (2014). Event-related Potentials (ERPs) in Second Language Research: A Brief Introduction to the Technique, a Selected Review, and an Invitation to Reconsider Critical Periods in L2. Applied Linguistics, 35(4), 393-417. https://doi.org/10.1093/applin/amu028 
Suomi, K., Toivanen, J., \& Ylitalo, R. (2003). Durational and tonal correlates of accent in Finnish. Journal of Phonetics, 31(1), 113-138. https://doi.org/10.1016/S0095-4470(02)00074-8

Tadel, F., Baillet, S., Mosher, J. C., Pantazis, D., \& Leahy, R. M. (2011). Brainstorm: A User-Friendly Application for MEG/EEG Analysis [Research Article]. Computational Intelligence and Neuroscience. https://doi.org/10.1155/2011/879716

Tamminen, J., Davis, M. H., Merkx, M., \& Rastle, K. (2012). The role of memory consolidation in generalisation of new linguistic information. Cognition, 125(1), 107-112. https://doi.org/10.1016/j.cognition.2012.06.014

Tamminen, J., Davis, M. H., \& Rastle, K. (2015). From specific examples to general knowledge in $\begin{array}{lllll}\text { language learning. } & \text { Cognitive } & \text { Psychology, } & 79, & \text { 1-39. }\end{array}$ https://doi.org/10.1016/j.cogpsych.2015.03.003

Taulu, S., \& Simola, J. (2006). Spatiotemporal signal space separation method for rejecting nearby interference in MEG measurements. Physics in Medicine and Biology, 51(7), 1759-1768. https://doi.org/10.1088/0031-9155/51/7/008

Tesche, C. D., Uusitalo, M. A., Ilmoniemi, R. J., Huotilainen, M., Kajola, M., \& Salonen, O. (1995). Signal-space projections of MEG data characterize both distributed and well-localized neuronal sources. Electroencephalography and Clinical Neurophysiology, 95(3), 189-200. https://doi.org/10.1016/0013-4694(95)00064-6

Turken, A. U., \& Dronkers, N. F. (2011). The Neural Architecture of the Language Comprehension Network: Converging Evidence from Lesion and Connectivity Analyses. Frontiers in System Neuroscience, 5. https://doi.org/10.3389/fnsys.2011.00001

Tyler, L. K., deMornay-Davies, P., Anokhina, R., Longworth, C., Randall, B., \& Marslen-Wilson, W. D. (2002). Dissociations in Processing Past Tense Morphology: Neuropathology and Behavioral Studies. Journal of Cognitive Neuroscience, 14(1), 79-94. https://doi.org/10.1162/089892902317205348

Ullman, M. T. (2004). Contributions of memory circuits to language: The declarative/procedural model. Cognition, 92(1), 231-270. https://doi.org/10.1016/j.cognition.2003.10.008

Ullman, M. T. (2006). Is Broca's area part of a basal ganglia thalamocortical circuit? Cortex; a Journal Devoted to the Study of the Nervous System and Behavior, 42(4), 480-485. https://doi.org/10.1016/s0010-9452(08)70382-4

Ullman, M. T. (2016). Chapter 76 - The Declarative/Procedural Model: A Neurobiological Model of Language Learning, Knowledge, and Use. In G. Hickok \& S. L. Small (Eds.), Neurobiology of Language (pp. 953-968). Academic Press. https://doi.org/10.1016/B978-0-12-4077942.00076-6

Vannest, J., Bertram, R., Järvikivi, J., \& Niemi, J. (2002). Counterintuitive Cross-Linguistic Differences: More Morphological Computation in English Than in Finnish. 24.

Whiting, C. M., Marslen-Wilson, W. D., \& Shtyrov, Y. (2013). Neural dynamics of inflectional and derivational processing in spoken word comprehension: Laterality and automaticity. Frontiers in Human Neuroscience, 7. https://doi.org/10.3389/fnhum.2013.00759

Yue, J., Bastiaanse, R., \& Alter, K. (2014). Cortical plasticity induced by rapid Hebbian learning of novel tonal word-forms: Evidence from mismatch negativity. Brain and Language, 139, 10-

Supplementary Material 22. https://doi.org/10.1016/j.bandl.2014.09.007

Comparison of neural source responses to the diminutive suffix "-ke" and the interrogative suffix "$k o$ " in the time windows of interest. Greenhouse-Geisser-corrected $F$ and $p$ values are reported if the assumption of sphericity was violated based on Mauchly's Test.

1. $130 \mathrm{~ms}$

Stem main effect: $F(1,18)=.212, p=.651$

Suffix main effect: $F(1,18)=.187, p=.671$

ROI main effect: $F(1.63,29.30)=39.239, p<.001$

Stem $x$ Suffix interaction: $F(1,18)=.003, p=.958$ 
Stem $x$ ROI interaction: $F(3,54)=.433, p=.730$

Suffix $x$ ROI interaction: $F(3,54)=.235, p=.872$

Stem $x$ Suffix $x$ ROI interaction: $F(1.60,28.84)=.478, p=.583$

2. $240 \mathrm{~ms}$

Stem main effect: $F(1,18)=1.998, p=.175$

Suffix main effect: $F(1,18)=.418, p=.526$

ROI main effect: $F(1.35,24.27)=11.037, p<.001$

Stem $x$ Suffix interaction: $F(1,18)=.071 p=.793$

Stem $x$ ROI interaction: $F(3,54)=.335, p=.800$

Suffix $x$ ROI interaction: $F(2.34,42.16)=.094, p=.934$

Stem $x$ Suffix $x$ ROI interaction: $F(1.58,28.40)=1.276, p=.288$ 


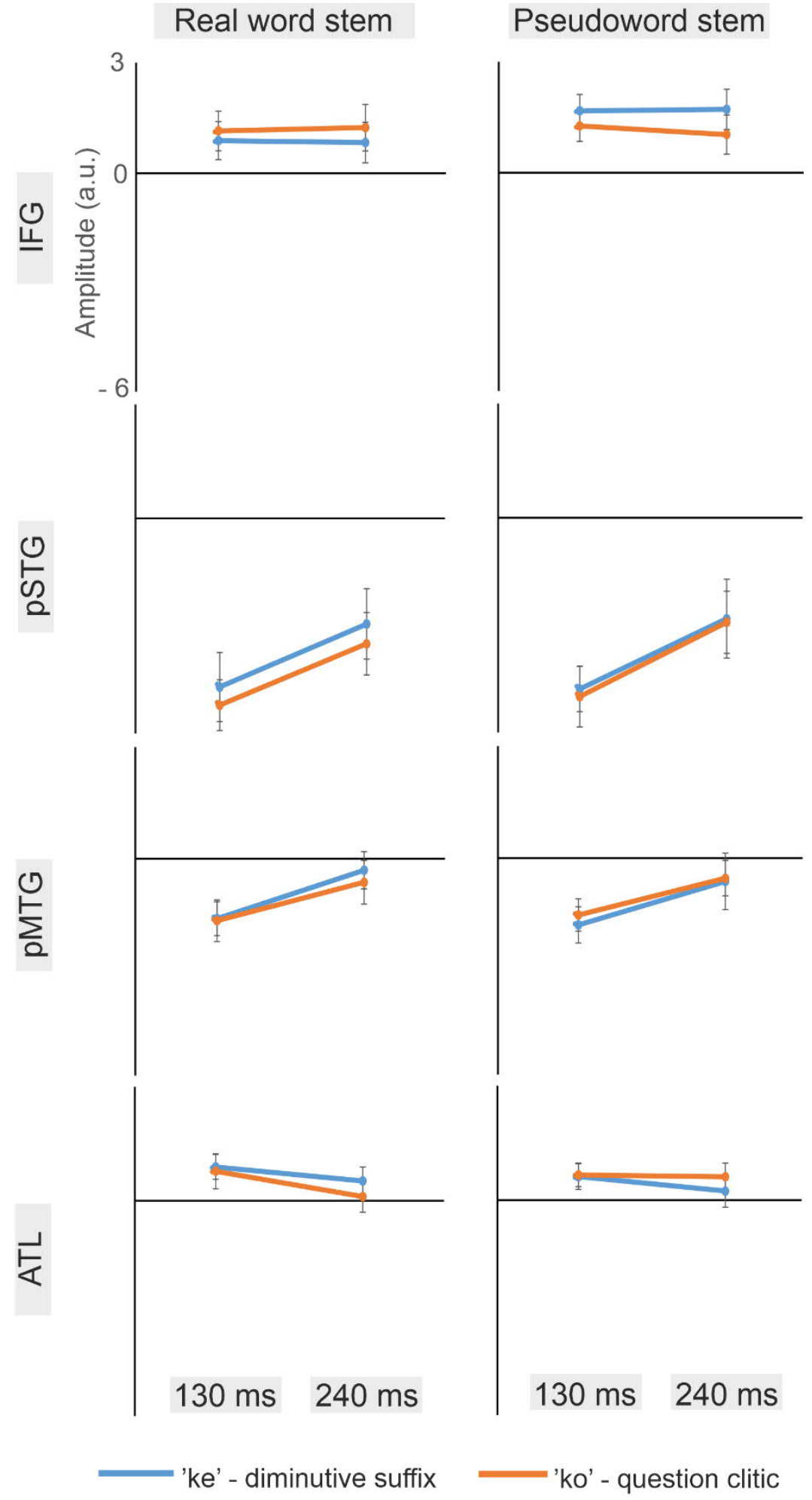

Supplementary Fig. 1. Neural source responses to the diminutive suffix "-ke" and the question clitic "ko" showed no significant differences in the time windows of interest. Activation is shown in arbitrary units (a.u.) based on the zscore normalization of the current density maps. The error bars mark the standard error. 